\title{
Das Capgras-Syndrom oder die Suche nach dem Ich
}

\author{
Erhard Taverna
}

Das Syndrom wurde erstmals 1923 vom französischen Psychiater Jean Marie Joseph Capgras beschrieben. Seine Patienten konnten Personen und Tiere, denen sie emotional verbunden waren, nicht mehr erkennen, obwohl der Teil des Gehirns, der Gesichter erkannte, so intakt war wie das Gedächtnis. Die Erkrankten glaubten, dass Leute, die ihnen nahestanden, durch genau gleich aussehende Kopien von Robotern, Doppelgängern oder Ausserirdischen ersetzt worden waren. Deren Gesichter weckten Erinnerungen, aber keine Gefühle. Dieser Mangel an emotionaler Bestätigung wog schwerer als die rationale Gewissheit und führte oft zu Verfolgungswahn mit einer erhöhten Gewaltbereitschaft. Dabei erfand die Vernunft aufwendige und komplizierte Erklärungsversuche, um mit dieser traumatisierenden Kränkung umzugehen.

Der erfolgreiche amerikanische Autor Richard Powers, geboren 1957, beschreibt in seinem neuen Roman «Das Echo der Erinnerung» die Geschichte eines verunfallten jungen Arbeiters, der nach einer langwierigen Rehabilitation nicht mehr fähig ist, seine eigene Schwester zu erkennen. Zwar anerkennt er nach und nach ihre Fürsorge und Pflege und lernt auch zu akzeptieren, dass sie mehr über seine Vergangenheit weiss als er selber, doch für ihn bleibt sie eine Ersatzschwester. Das Thema Hirnforschung hat spätestens seit Oliver Sacks und Antonio Damasio auch Eingang in die Literatur gefunden. Ihre Ergebnisse sind das Material zeitgemässer Schauplätze für die alten Sinnfragen. Der Autor verknüpft das Schicksal mehrerer Bezugspersonen des Unfallopfers zu einer spannenden Handlung, in der medizinische und ökologische Probleme im Brennpunkt stehen. Neuronale Vorgänge und Forschungsergebnisse sind anschaulich und lehrreich in Sprache umgesetzt. In Frage gestellt werden die fragile Existenz der Selbstgewissheit, die Mechanismen der Selbsterhaltung und die wichtigsten Merkmale des «Ich», wie Einheit, Willensfreiheit, Verkörperung, Kontinuität und Fähigkeit der Reflexion. Was wie eine interessante Fallgeschichte beginnt, entwickelt sich zur überlebenswichtigen Selbstsuche von Patient und Arzt. Ausgerechnet in der tiefsten Provinz von Nebraska, in einer Kleinstadt, «wo das Inter-

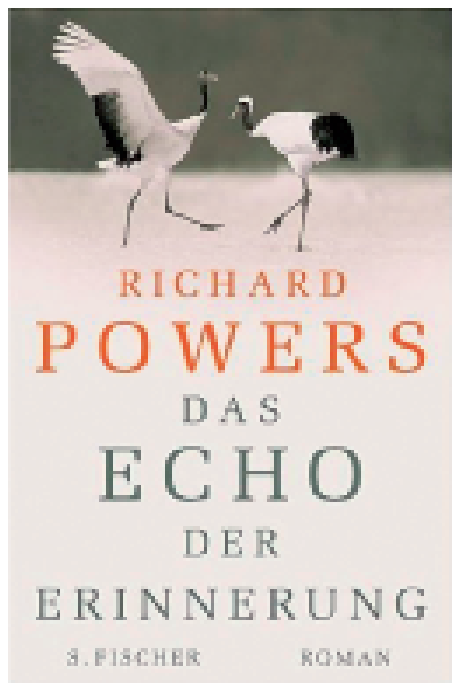

net wie Schnaps über einen Steinzeitstamm gekommen war», in einem langweiligen Kaff, das nur für kurze Zeit als Rastplatz riesiger Kranichschwärme bekannt ist, gerät der berühmte Schriftsteller und Konsiliarneurologe in seine tiefste Lebenskrise. Mit der Hypothese einer gestörten Verbindung zwischen intakter Amygdala und unversehrtem Inferotemporalkortex mag er sich nicht zufriedengeben: «Was zählt, ist also nicht das Wissen um ein Gefühl, sondern das gefühlte Wissen.» Natürlich kennt er das Phänomen der persönlichen Konfabulation, der Konstruktion einer Geschichte, mit deren Hilfe das verstörte Ich ein neues Verhältnis zu unverständlichen Fakten herstellt. Jedes Gehirn produziert vernünftige Erklärungen für ungewöhnliche Wahrnehmungen. Doch was der Arzt bisher immer nur seinen Patienten zuschrieb, erkennt er als eigene Existenzbedingung: «Niemand hatte ein Ich, das so aussah, wie das Ich sich selbst definierte. Lügen, Selbsttäuschung und Verdrängung waren keine Krankheitssymptome, sie waren Ergebnis der Reflexion. Als Krönung des Ganzen erfand das Gehirn ein Ich.» Die Erkenntnisse Freuds holen ihn endlich ein. Nach einer schlechten Buchkritik bricht ein lange verborgener Selbstzweifel in ihm auf. Seine bisherige Überzeugung, wonach Geschichten Wirklichkeit wurden: «wir erzählen uns zurück zur Diagnose und vorwärts in die Therapie», wird 
massiv erschüttert. In der neuen klinischen Welt der bildgebenden Verfahren und molekularen Hirnforschung kommt er sich plötzlich als nutzloser Anekdotenerzähler vor. Seine auflagenstarken Fallgeschichten und die bei Studenten populären Vorlesungen sind ihm nur noch Zirkusnummern. Richard Powers erzählt die verzweifelte Sinnsuche seiner Protagonisten vor dem Hintergrund eines rätselhaften Schauspiels von Hunderttausenden Kranichen, die sich seit Millionen von Jahren auf der Wanderung nach Norden jährlich am Fluss einfinden. In diesem natürlichen Kreislauf findet der Arzt Trost vor der eigenen trügerischen und vergänglichen Gegenwart. Im komplexen Erinnerungsvermögen dieser Vögel erkennt er die Wurzeln der eigenen Existenz, «denn sein Hirnstamm ist noch immer eine Zuflucht für ältere Verwandte». Die Kraniche sind für ihn den Menschen überlegen, denn ihre zeitlose Orientierung braucht nicht die trügerischen und kurzlebigen Tricks des menschlichen Gehirns. Die Zukunft der Erde kommt ohne Menschen aus: «Das Aussterben geht schnell, doch die Wanderung ist lang. Die Natur und ihre Landkarten werden selbst das Schlimmste nutzen, was die Menschheit ihnen antun kann.»

Naturmystik im Zeichen von PET und MRI? Der Roman ist trotz Schwächen lesenswert, weil er zu einer verstörenden Einsicht kommt: Die Wissenschaft weiss keine Antwort für einen Menschen, der nicht mehr an seine Erzählungen glaubt. Er muss sich neu erfinden. Wem der Boden wegbricht, dem bleibt nichts anderes übrig, als eine neue Geschichte zu erzählen.

- Richard Powers. Das Echo der Erinnerung. Frankfurt am Main: S. Fischer Verlag; 2006. 533 Seiten.

\section{Die Abmagerungskur oder wie ich fast Anorexia wurde}

Peter Marko

Korrespondenz:

Dr. med. Peter Marko Bruggwaldstrasse 39e CH-9009 St. Gallen
«Du wirst, wie auch ich, nie dick werden», versicherte mir immer wieder mein Vater. Dreissigjährig, sprang ich mit nicht ganz siebzig Kilo über den Eisernen Vorhang. Auf der anderen Seite gelandet, stillte ich während der nächsten Jahre meine Neugier und mein Bedürfnis nach all den guten, bisher nicht gekannten Speisen oder solchen, die man sich finanziell nicht erlauben konnte, wie verschiedenen Käse- und Fleischsorten, Schokolade, Bananen, und brachte bald achtzig Kilo auf die Waage. Während der Ferienreisen lernten wir neue Speisen kennen, gern haben (meistens ich) und zubereiten (nur meine Frau). So begann ich nach dem Englandbesuch den Tag nicht selten mit Speck und Eiern. Wenn man schon im täglichen Leben stark belastet ist, sollte es an gutem Essen nicht fehlen, oder? Ich bekam so oft wie möglich meine Lieblingsgerichte aufgetischt, und nach jedem Mittagessen durfte ein Dessert nicht fehlen. Zur Entspannung klang der Abend mit einem Glas Bier oder Wein aus. In den Restaurants suchten wir möglichst erlesene Speisen aus.

Meine Hemden, Jacken, Hosen wurden dauernd etwas enger. Klar, mit dem Alter richten sich die Rippen auf, und der Brustkorb verbreitert sich dadurch. Meine Frau machte mich ab und zu darauf aufmerksam, dass mir das Fett über den Gurt zu quellen beginne. Eben, auch die Haut wird weniger elastisch und bildet leichter Wulste. Als ich ihn auf dem Rücken liegend betrachtete, war der Bauch aber doch flach auf der Ebene des Brustbeines. Ich meinte, es sei nur Sache des Standpunktes, und beruhigte sie, oder mich, mit den Worten meines Vaters, die ich anfangs erwähnte.

Es war immer mühsamer, in der Zeitnot schnell zu den Zügen zu laufen, und etliche Male fuhr mir das Tram vor der Nase weg. Nach dem Restaurantbesuch während der Ferien kletterte ich in unsere Wohnung mit schwerem Atem und nötigen Pausen den Berg hinauf und wünschte, die Lage wäre umgekehrt, ich könnte zuerst mit leerem Magen hinauf ins Restaurant laufen und dann mit vollem, rundem Leib leichter runter in die Wohnung kugeln. Offensichtlich meldete sich bei mir schon die Altersherzschwäche. Als ich die Skistiefel schliessen musste, konnte ich mich, nicht wegen Rückenschmerzen, kaum biegen, und mein Freund musste immer wieder warten, bis ich ihm am Hang folgen konnte. Aus den letzten Skiferien nach Hause zurückgekehrt, wog ich zweiundneunzig Kilo. Jetzt reichte es mir, es musste etwas geschehen! 
Ich begann regelmässig, fünf- bis sechsmal pro Tag zu essen, damit sich der Hunger nie meldete, da er ähnlich dem Alkohol den Willen bricht und den Weg zum Ziel erschwert. Als Zwischenmahlzeiten nahm ich Obst, rohes Gemüse (Kohlrabi, Karotten, Radieschen, Blumenkohl schmeckt vorzüglich, und ich bekam dadurch weder grosse Ohren noch Hasenzüge) oder verschiedene Getreidewaffeln und -fladen. Ich ass auch nur so viel, wie nötig war, den Hunger vom Leibe zu halten, und nicht wieviel ich mochte. Damit gewöhnte ich mich leichter an die neuen einfachen, «reinen» Speisen und begann wieder ihren guten Geschmack zu schätzen. Eigentlich alle die Beilagen, Sossen, Mayonnaisen und raffinierten Gewürzmischungen verdarben ihn nur. Nach einem Monat war ich bereits um drei Kilo leichter. Mit den verlorenen Kilos fühlte ich mich immer wohler. Ich kann mich beherrschen, das Ziel verfolgen, mein Geist kann die niedrigen Gelüste des Körpers besiegen, kurzum, ich bin ein starker, erfolgreicher Mensch. Ich fühlte mich wie ein Hobbygärtner - was immer ihm auch beruflich und persönlich zustösst, die Pflanzen, die er gesetzt hat, gedeihen und blühen, und er sieht, schmeckt und geniesst die Früchte seiner Bemühung (ausser, wenn ihm Hagel alles zerstört!). Bei verschiedenen Gelegenheiten beobachtete ich mit gewisser Erhabenheit die eher rundlichen Gestalten neben mir, die plan- und hemmungslos dick belegte Brote, schuhsohlengrosse Steaks, phantasievolle Desserts in sich hineinstopften, genüsslich mampften und alles mit Wein runterspülten und so ihre Gesundheit langsam, aber sicher, ob bewusst oder unbewusst, ruinierten, während ich zum fast heiligen Zweck an meinem Glas reinen Mineralwassers nippte.

Nach einem Jahr verschwanden die Fettwülste, der Bauch war auch stehend auf dem Niveau des Brustbeines, liegend machte er sogar eine Delle, ich bekam wieder Taille, lief den «StutZ» aus dem Restaurant in die Ferienwohnung trotz vollem Magen wie ein Reh hinauf, und der Freund musste beim Skifahren darauf achten, dass ich die Piste nicht als erster runtersauste.

Die lästigen Erkältungsviren, die mir jahrelang während des Winters fast ununterbrochen das Leben erschwerten, machten plötzlich einen weiten Bogen um mich, der Blutdruck war auch ohne Betablocker immer im Normbereich, meine Gicht hatte ich mit einem Bruchteil der bisherigen Medikamentendosis unter Kontrolle, beim Belastungstest erreichte ich überdurchschnittliche Werte und auf der Waage fünfundsiebzig Kilo. Ich durfte zufrieden sein, oder? Das reichte mir aber nicht, ich wollte das Gewicht meiner Jugend haben, wie die von mir bewunderten Tenniscracks aussehen.

Jetzt begann ich mich zu plagen. Ich ass nur noch kalorienarme, vorwiegend aus Zelluloseund anderen Fasern bestehende Speisen, also fast nur Gemüse und Obst, und schwamm ein, zwei Kilometer pro Tag, manchmal bei siebzehn Grad Wassertemperatur, aber ausser einem unerträglichen Hunger erreichte ich nicht viel. Ich sah zwar immer noch nicht wie ein Hase aus, aber offensichtlich begann ich allmählich wie ein Ochse sogar die Zellulose zu verwerten. Schweren Herzens begrub ich den Traum und steckte den Misserfolg ein. Auch das Anti-Aging hat seine Grenzen. 